\title{
Evaluación de los efectos cardiorrespiratorios del butorfanol adjunto a un protocolo de anestesia total intravenosa en cabras sometidas a laparoscopia
}

\author{
Evaluation of cardiorespiratory effects of an adjunct butorphanol in total intravenous \\ anaesthesia protocol in goats under laparoscopy
MF Cordeiro ${ }^{a}$, RAR Uscategui ${ }^{b *}$, MEF Oliveira ${ }^{b}$, DPM Dias $^{c}$, PA Di Filippo ${ }^{\mathrm{d}}$, WAS Restan ${ }^{\mathrm{b}}$, CAA Valadão ${ }^{\mathrm{b}}$, LA Coelho ${ }^{\mathrm{e}}$, WRR Vicente ${ }^{\mathrm{b}}$

\begin{abstract}
To evaluate the cardiorespiratory effects and postoperative analgesia of a total intravenous anesthesia protocol, twenty-four adult goats were assigned to four experimental groups: G1 and G2 underwent to laparoscopic procedure every 15 days, while G3, and G4 every 30 days. Xylazine $(0.05 \mathrm{mg} / \mathrm{kg} \mathrm{IM})$ in all animals and butorphanol $(0.5 \mathrm{mg} / \mathrm{kg} \mathrm{IM})$ in animals of G2 and G4 were used as premedication. Ketamine $(2.0 \mathrm{mg} / \mathrm{kg} \mathrm{IV})$ anaesthetic induction was performed and subsequently the sheep were intubated and received oxygen $\left(\mathrm{FiO}_{2}=1.0\right)$. Animals were positioned in a Trendelenburg position and anaesthetic plane maintained by constant infusion of $2 \mathrm{~mL} / \mathrm{kg} / \mathrm{h} \mathrm{IV}$ of a solution composed of: $12.5 \mathrm{~g}$ of guaiacolate, $25 \mathrm{mg}$ of xylazine and $250 \mathrm{mg}$ of ketamine in $250 \mathrm{~mL}$ of $5 \%$ dextrose. Haemo-gasometric ( $\mathrm{pH}, \mathrm{PO}_{2} \mathrm{PCO}_{2}, \mathrm{CHCO}_{3}$ ) and clinical parameters (HR, RR, MAP and T), recovery time and postoperative analgesia were evaluated at different times of the anesthetic-surgical procedure. $\mathrm{HR}, \mathrm{RR}, \mathrm{T}, \mathrm{pH}, \mathrm{PO}_{2}, \mathrm{PCO}_{2}$ and $\mathrm{cHCO}_{3}$ varied through surgical time and $\mathrm{PCO}_{2}$ was significantly higher in butorphanol treated animals. The studied anaesthetic protocol resulted in a prolonged recovery, clinical hypotension and respiratory acidosis; butorphanol supplementation caused an increase in PaCO2 with no evident improvement of postoperative analgesia. Therefore, the practical application of this protocol indicates a special fluid, ventilation and recovery care. Further studies are needed on the analgesic effect of butorphanol in these situations to allow its indication.

Keywords: butorphanol, xylazine, ketamine, hypotension.
\end{abstract}

RESUMEN. Para evaluar los efectos cardiorrespiratorios y la analgesia posoperatoria de un protocolo de anestesia total intravenosa fueron utilizadas 24 cabras adultas, distribuidas en cuatro grupos experimentales: G1 y G2, sometidos a laparoscopia cada 15 días, G3 y G4 cada 30 días. Como medicación preanestésica se utilizaron $0,05 \mathrm{mg} / \mathrm{kg}$ IM de xilacina en todos los animales y $0,5 \mathrm{mg} / \mathrm{kg} \mathrm{IM} \mathrm{de}$ butorfanol en los grupos G2 y G4. La inducción anestésica fue realizada con ketamina (2,0 mg/kg IV), se realizó la intubación traqueal y administración de oxígeno $\left(\mathrm{FiO}_{2}=1,0\right)$. Los animales fueron posicionados en Trendelemburg y mantenidos en plano anestésico mediante infusión continua de $2 \mathrm{~mL} / \mathrm{kg} / \mathrm{h}$ de una solución compuesta por $12,5 \mathrm{~g}$ de guayacolato, $25 \mathrm{mg}$ de xilacina y $250 \mathrm{mg}$ de ketamina, diluidos en $250 \mathrm{~mL}$ de dextrosa al 5\%. Parámetros hemogasométricos ( $\mathrm{pH}, \mathrm{PaO}_{2} \mathrm{PaCO}_{2}, \mathrm{cHCO}_{3}$ ), clínicos (FC, FR, PAM y T), tiempo de recuperación y analgesia posoperatoria fueron evaluados en diferentes momentos del procedimiento anestésico-quirúrgico. La FC, FR, T, pH, $\mathrm{PaO}_{2}, \mathrm{PaCO}_{2}$ y cHCO 3 variaron con relación al tiempo quirúrgico y la $\mathrm{PaCO}_{2}$ fue significativamente mayor en los animales en los que fue utilizado butorfanol. Con este protocolo anestésico se observó un tiempo de recuperación prolongado, hipotensión clínica y acidosis respiratoria; la suplementación con butorfanol resultó en un aumento de la $\mathrm{PaCO}_{2}$ sin mejora evidente de la analgesia posoperatoria. Así, la aplicación práctica de este protocolo indica un cuidado especial de la fluidoterapia, la ventilación y la recuperación. Estudios más profundos del efecto analgésico del butorfanol en estas situaciones se hacen necesarios para permitir indicar su utilización.

Palabras clave: butorfanol, xilacina, ketamina, hipotensión.

\section{INTRODUCCIÓN}

La anestesia total intravenosa (TIVA por sus siglas en inglés) consiste en la administración de varios fármacos inyectables con el objetivo de proporcionar analgesia, pérdida de la conciencia, amnesia, relajación muscular

Aceptado: 29.10.2015.

aUniversidade Federal do Vale do São Francisco, Petrolina, PE, Brasil.

bFaculdade de Ciências Agrarias e Veterinárias, Universidade Estadual Paulista, Jaboticabal, SP, Brasil.

${ }^{\mathrm{c} C e n t r o ~ U n i v e r s i t a ́ r i o ~ B a r a ̃ o ~ d e ~ M a u a ́, ~ R i b e i r a ̃ o ~ P r e t o, ~ S P, ~ B r a s i l . ~}$

${ }^{\mathrm{d} C}$ Centro de Ciências e Tecnologias Agropecuárias, Universidade Estadual do Norte Fluminense, Goytacazes, RJ, Brasil.

eFaculdade de Medicina Veterinária e Zootecnia, Universidade de São Paulo, Pirassununga, SP, Brasil.

*Autor de correspondencia: RAR Uscategui; Via de Acesso Professor Paulo Donato Castellani, s/n, 14884-900 Jaboticabal, SP, Brasil; ramirezuscategui@hotmail.com y manutención de las funciones autonómicas; que permitan la realización del procedimiento quirúrgico con mínimas alteraciones fisiológicas. Esta técnica no exige la utilización de equipamientos para la administración de anestésicos inhalatorios y puede ser más práctica a campo. Sin embargo, el control de la profundidad anestésica es más difícil y la recuperación generalmente es prolongada (Clarke y col 2014).

La aspiración folicular por laparoscopia (LOPU) es un procedimiento quirúrgico que requiere la aplicación de técnicas anestésicas en pequeños rumiantes. Se considera mínimamente invasiva, permite reducir el trauma tisular, proporcionando menor convalecencia y dolor posoperatorio (Malm y col 2004). Sin embargo, esta técnica requiere el posicionamiento del animal en decúbito dorsal, la instauración de neumoperitoneo con $\mathrm{CO}_{2}$ y la posición de Trendelenburg, que pueden ocasionar alteraciones fisiológicas importantes, como disminución del retorno 
venoso, de la capacidad pulmonar residual, de la complacencia pulmonar y de los flujos renal, esplénico y portal; también incrementan la poscarga, la resistencia vascular sistémica (RVS), la presión arterial media (PAM) y la presión intracraneal, resultando en detrimento del débito cardiaco proporcional a la presión abdominal instaurada. Por otro lado, el $\mathrm{CO}_{2}$ utilizado para la insuflación abdominal puede ser absorbido y generar hipercapnia. La respuesta neurohormonal a todas estas alteraciones es la activación del sistema simpático y la liberación de catecolaminas y vasopresina (Schiller 1987, Bardoczky y col 1993, Fahy y col 1996, O'Leary y col 1996, O’Leary y col 1996).

A pesar de ser el abordaje mínimamente invasivo, puede ocasionar dolor en los animales, este signo prolonga la recuperación, retarda la cicatrización, deprime el sistema inmunológico, perjudica la homeostasis corporal y aumenta el metabolismo y catabolismo tisular (Otero 2005). El control intraoperatorio del dolor es importante, pues la anestesia general no elimina los procesos neuronales periféricos y centrales responsivos a la nocicepción (Johnston 2005).

Los protocolos anestésicos balanceados para este tipo de procedimientos han sido pobremente estudiados en pequeños rumiantes (LiJian y col 2008, Tekin y col 2009), $\mathrm{y}$ algunos autores indican al butorfanol como un opioide seguro y efectivo para su utilización en caprinos (Carroll y col 1998, Dzikiti y col 2009). No obstante, ninguno de estos trabajos comparó los efectos fisiológicos de los procedimientos repetidos en un corto intervalo de tiempo, ni del tratamiento analgésico opioide conjunto. Con lo expuesto anteriormente, el objetivo de esta investigación fue evaluar los efectos sobre el sistema cardiorrespiratorio, los gases sanguíneos, la recuperación anestésica y la analgesia posoperatoria de un protocolo de TIVA, utilizando o no butorfanol, para la LOPU repetida a cada 15 o 30 días en cabras.

\section{MATERIAL Y MÉTODOS}

Fueron utilizadas 24 hembras caprinas mestizas, adultas, saludables, que nunca fueron sometidas anteriormente a procedimientos quirúrgicos y con un peso medio de 27,9 $\pm 6 \mathrm{~kg}$. Estos animales se mantuvieron en estabulación, recibiendo una dieta balanceada y acceso a agua potable ad libitum, desde un mes antes del procedimiento y hasta el final del periodo experimental. Las hembras fueron aleatoriamente distribuidas en cuatro grupos experimentales de igual número $(n=6)$ de la siguiente forma: grupos 1 y 2 (G1 y G2) sometidas a aspiración folicular por laparoscopia cada 15 días, y grupos 3 y 4 (G3 y G4) cada 30 días. Los grupos $\mathrm{G} 2$ y $\mathrm{G} 4$ recibieron tratamiento opioide.

Después de un ayuno de sólidos de 48 horas y líquidos de 24 horas fue aplicada como medicación preanestésica $0,05 \mathrm{mg} / \mathrm{kg}$ de clorhidrato de xilacina ${ }^{1}$ por vía intramuscular (IM) en todos los animales, y $0,5 \mathrm{mg} / \mathrm{kg}$ IM de tartarato

1 Dorcipec®, Vallée, Montes Claros-MG, Brasil. de butorfanol ${ }^{2}$ en los grupos G2 y G4. A continuación, un catéter heparinizado (22G) fue colocado en la arteria auricular caudal para colecta de sangre arterial y monitorización continua de la presión arterial y un catéter (16G) en la vena yugular para la administración de fármacos y fluidos. Diez minutos después fue administrado por vía intravenosa (IV), clorhidrato de ketamina ${ }^{3}$ en dosis de $2,0 \mathrm{mg} / \mathrm{kg}$, se realizó la intubación orotraqueal (sonda de Maguil 7,5 D con manguito) y la administración de oxígeno $\left(\mathrm{FiO}_{2} 1.0\right)$. Las hembras fueron colocadas en la camilla para aspiración folicular laparoscópica y se inició la infusión continua IV, de la solución anestésica compuesta por $12,5 \mathrm{~g}$ de guayacol gliceril éter ${ }^{4}, 25 \mathrm{mg}$ de clorhidrato de xilacina y $250 \mathrm{mg}$ de clorhidrato de ketamina, diluidos en $250 \mathrm{~mL}$ de solución de dextrosa al $5 \%$, a una taza de infusión inicial de $2 \mathrm{~mL} / \mathrm{kg} / \mathrm{h}$ ajustándola al plano anestésico por medio de una bomba de infusión peristáltica 5 .

En este momento fue realizada la preparación aséptica de la zona quirúrgica (región desde el manubrio esternal hasta la ubre) con yodopovidina y la infiltración dérmica y muscular en los lugares de punción, de $2 \mathrm{~mL}$ de clorhidrato de lidocaína al $2 \%{ }^{6}$. Los animales se posicionaron en Trendelenburg (decúbito dorsal con la cabeza para abajo en un ángulo de inclinación de $45^{\circ}$ ). La técnica quirúrgica fue realizada de la misma forma en todos los animales, empleando 2 portales, insuflación abdominal con $\mathrm{CO}_{2}$ a $8 \mathrm{mmHg}$ de presión y punción transabdominal de los folículos ováricos con el mandril de un catéter $18 \mathrm{G}$. El tiempo de laparoscopia fue establecido en 40 minutos, realizado siempre por el mismo cirujano y repetido 4 veces en todos los animales a diferentes intervalos según el grupo experimental (15 o 30 días). Una vez finalizado el procedimiento y retirado el $\mathrm{CO}_{2}$ del abdomen, la infusión de TIVA fue suspendida y los animales fueron posicionados en decúbito lateral, recibiendo oxígeno durante 15 minutos más para, posteriormente, reposicionarlos en decúbito esternal, hasta la recuperación del reflejo de deglución para poder retirar la sonda orotraqueal. Como tratamiento postoperatorio fue aplicada la combinación de penicilina $\mathrm{G}$ (potásica, procaínica y benzatínica, $15000 \mathrm{UI} / \mathrm{kg}$ ), estreptomicina $(10 \mathrm{mg} / \mathrm{kg})$ y diclofenaco sódico ${ }^{7}$ (1 mg/ $\mathrm{kg}$ ). Posteriormente los animales fueron llevados a una pesebrera individual de recuperación hasta que pudieran alimentarse normalmente para ser liberados en el corral de experimentación con sus congéneres.

\footnotetext{
2 Torbugesic ${ }^{\circledR}$, Fort Dodge, Campinas-SP, Brasil.

3 Dopalen ${ }^{\circledR}$, Vetbrands, Jacareí-SP, Brasil.

4 Guaifenesina ${ }^{\circledR}$, Henrifarma, São Paulo-SP, Brasil.

5 ST550T2, Samtronic $®$, São Paulo-SP, Brasil.

6 Lidovet@, Bravet, Rio de Janeiro-RJ, Brasil.

7 Penfort@ Reforçado, Ourofino, Cravinhos, SP, Brasil.
} 
Las muestras de sangre arterial fueron colectadas en jeringas heparinizadas de $1 \mathrm{~mL}$ y herméticamente selladas, identificadas con la temperatura corporal del momento de colecta y procesadas inmediatamente para su análisis gasométrico arterial automatizado ${ }^{8}$, midiendo el potencial de hidrógeno $(\mathrm{pH})$, presión parcial de oxígeno en $\mathrm{mmHg}$ $\left(\mathrm{PaO}_{2}\right)$, presión parcial de dióxido de carbono en $\mathrm{mmHg}$ $\left(\mathrm{PaCO}_{2}\right)$, concentración de bicarbonato en $\mathrm{mmol} / \mathrm{L}\left(\mathrm{cHCO}_{3}\right)$, en los momentos: (M0) 10 minutos después de la medicación preanestésica; (M1) inmediatamente después de la intubación; (M2) inmediatamente después de la insuflación abdominal en Trendelenburg; (M3) 15 minutos después de M2 y (M4) 15 minutos después de retirada la TIVA. En estos momentos los parámetros clínicos de frecuencia cardiaca en lat/min (FC), frecuencia respiratoria ciclos/ min (FR), presión arterial media en mmHg (PAM) y temperatura rectal en ${ }^{\circ} \mathrm{C}(\mathrm{T})$ fueron evaluados y anotados con la ayuda de un monitor multiparámetros ${ }^{9}$.

El tiempo en minutos transcurrido desde el cese de la infusión anestésica hasta recuperar la posición cuadrupedal espontáneamente fue considerado como el tiempo de recuperación anestésica. Estos animales fueron observados en el corral experimental 2, 4, 6, 12, 24, 36, 48, 60 y 72 horas después del procedimiento quirúrgico por dos evaluadores entrenados para identificar alteraciones comportamentales que pudieran indicar dolor, como pérdida de movilidad, vocalización, actividades motoras repetidas, ausencia de socialización, permanencia en decúbito lateral o inapetencia ${ }^{10}$. Si alguno de estos signos clínicos era observado se administraba medicación analgésica de rescate con clorhidrato de tramadol ${ }^{11}$ (4 mg/kg IV).

$\mathrm{El}$ análisis estadístico fue realizado con la ayuda del software $\mathrm{R}{ }^{12}{ }^{12}$. Los parámetros estudiados fueron comparados entre los grupos y momentos por medio del análisis de varianzas (ANOVA) previa comprobación de los supuestos matemáticos, en un diseño experimental factorial (AxB), donde el factor A correspondía al tratamiento opioide y el factor $\mathrm{B}$ al intervalo quirúrgico, con medidas repetidas en el tiempo. La significancia fue fijada en $95 \%(\mathrm{P}<0,05)$.

\section{RESULTADOS Y DISCUSIÓN}

Los parámetros cardiorrespiratorios evaluados en el presente estudio se encontraron dentro de los valores normales para la especie en anestesia general (FC 60-120 lat/min; PAM 65-95 mmHg; FR 15-30 ciclos/min), con

8 OMNI C@, Roche Diagnostics, 2858, São Paulo-SP, Brasil.

9 DX2010®, Dixtal Philips Healthcare, Barueri, Brasil.

10 George LW. 2003. Pain Control in Food Animals. In: Steffey EP (ed). Recent Advances in Anesthetic Management of Large Domestic Animals. Disponible en www.ivis.org.

11 Tramadon®, Cristalia, São Paulo, Brasil.

12 R Foundation for Statistical Computing, Vienna, Austria. excepción de la PAM que fue inferior a lo esperado $(58,03$ $\pm 0,84 \mathrm{mmHg}$ ), considerando que los animales presentaron hipotensión clínica durante el procedimiento anestésicoquirúrgico (Clarke y col 2014). Esta alteración se presentó desde la medicación preanestésica y puede ser atribuida al protocolo anestésico (M0 55,71 $\pm 1,94 \mathrm{mmHg}$ ), ya que la disminución de la presión arterial es un efecto dosisdependiente de la xilacina, así como de la asociación de este medicamento con la ketamina en pequeños rumiantes (Kokkonen y Eriksson 1987, Celly y col 1997, Afshar y col 2005). La FC fue mayor $(\mathrm{P}=0,01)$ después de la medicación preanestésica (74,6 $\pm 16,2$ lat/min), cuando se compara con el periodo anestésico $(64,9 \pm 14,5$ lat $/ \mathrm{min})$, esta alteración puede relacionarse con el estrés propio de la manipulación durante la preparación de los pacientes y con la utilización de la xilacina, una vez que este fármaco y su asociación con la ketamina presentan un efecto cronotrópico negativo dosis dependiente (Kokkonen y Eriksson 1987, Afshar y col 2005). Ningún efecto del butorfanol o del intervalo entre procedimientos fue evidente sobre la FC y la PAM (cuadro 1).

Durante M0 y M4, la FR fue menor $(\mathrm{P}<0,01)$ en relación con los demás momentos (cuadro 1); durante estas etapas, los animales se encontraban despiertos o en fase de recuperación anestésica, siendo este resultado correlacionado con la falta de estímulo doloroso y la ausencia de compresión diafragmática ocasionada por la posición de Trendelenburg y neumoperitoneo (Fahy y col 1996). La asociación de xilacina y ketamina en dosis clínicas no altera significativamente la frecuencia respiratoria según estudios realizados en caprinos (Kokkonen y Eriksson 1987, Afshar y col 2005), por este motivo y debido a los momentos de alteración, no puede ser indicada esta alteración como un efecto del protocolo anestésico. Con relación a $\mathrm{PaO}_{2}$, este parámetro aumentó significativamente $(\mathrm{P}<0,01)$ después de la intubación y administración de oxígeno a los animales, como era de esperarse debido al aumento de la $\mathrm{FiO}_{2}$ de 0,21 a 1,0, Clarke y col (2014) describieron que la $\mathrm{PaO}_{2}$ puede aumentar hasta 5 veces cuando los animales respiran $100 \%$ de oxígeno como en este caso. Aunque exista una mayor predisposición por parte de los rumiantes al colapso pulmonar debido al decúbito dorsal, a la posición de Trendelenburg y al neumoperitoneo (Safran y Orlando 1994, Clarke y col 2014), en este estudio la oxigenación arterial no sufrió alteraciones.

Durante el procedimiento anestésico fue evidenciada la alteración del equilibrio ácido básico. La disminución gradual del $\mathrm{pH}$ evidenciada en el tiempo $(\mathrm{P}<0,01)$ indica acidosis, que puede clasificarse como respiratoria una vez que la $\mathrm{PaCO}_{2}$ presentó un comportamiento similar $(\mathrm{P}<0,01)$ y la vía compensatoria (alcalosis metabólica) parece activada debido al aumento de $\mathrm{cHCO}_{3}(\mathrm{P}=0,03)$ (cuadro 1). Esta alteración es común durante cirugías laparoscópicas, siendo descrita en humanos, caninos y porcinos (Ivankovich y col 1975, Hayden y Cowman 2011, Güzel y col 2012) y puede ser consecuencia de 
Cuadro 1. Valores Medios ( $\pm \mathrm{EE}$ ) de los parámetros cardiorrespiratorios evaluados en cuatro diferentes momentos en cabras sometidas a laparoscopia, bajo anestesia total intravenosa en cuatro grupos de evaluación según el intervalo quirúrgico 15 (G1 y G2) o 30 (G3 y G4) días y la aplicación de butorfanol (G2 y G4).

Mean \pm SE of evaluated cardiorespiratory parameters at four different times in goats submitted to Laparoscopy under total intravenous anesthesia in four groups according to the surgical interval 15 days (G1 and G2) or 30 days (G3 and G4) and butorphanol treatment (G2 and G4).

\begin{tabular}{|c|c|c|c|c|c|c|}
\hline \multirow{2}{*}{ Parámetros } & \multirow{2}{*}{ Grupo } & \multicolumn{5}{|c|}{ Momento } \\
\hline & & M0 & M1 & M2 & M3 & M4 \\
\hline \multirow[t]{4}{*}{ FC } & G1 & $71,6 \pm 2,7 \mathrm{Aa}$ & $67,4 \pm 2,9 \mathrm{Ba}$ & $61,6 \pm 2,4 \mathrm{Ba}$ & $66,8 \pm 3,3 \mathrm{Ba}$ & $66,8 \pm 2,4 \mathrm{Ba}$ \\
\hline & $\mathrm{G} 2$ & $80,5 \pm 4,2 \mathrm{Aa}$ & $67,7 \pm 2,6 \mathrm{Ba}$ & $70,5 \pm 2,8 \mathrm{Ba}$ & $74,6 \pm 4,8 \mathrm{Ba}$ & $72,3 \pm 3,5 \mathrm{Ba}$ \\
\hline & G3 & $75,4 \pm 3,2 \mathrm{Aa}$ & $62,8 \pm 1,8 \mathrm{Ba}$ & $58,7 \pm 1,6 \mathrm{Ba}$ & $60,3 \pm 1,9 \mathrm{Ba}$ & $62,5 \pm 1,9 \mathrm{Ba}$ \\
\hline & G4 & $70,7 \pm 2,8 \mathrm{Aa}$ & $62,4 \pm 2,7 \mathrm{Ba}$ & $62,5 \pm 2,8 \mathrm{Ba}$ & $59,9 \pm 2,2 \mathrm{Ba}$ & $63,3 \pm 2,7 \mathrm{Ba}$ \\
\hline \multirow[t]{4}{*}{ FR } & G1 & $24,7 \pm 1,4 \mathrm{Aa}$ & $42,9 \pm 4,6 \mathrm{BCa}$ & $45,5 \pm 3,2 \mathrm{Ca}$ & $42,9 \pm 2,9 \mathrm{Ca}$ & $30,8 \pm 2,9 \mathrm{Ba}$ \\
\hline & $\mathrm{G} 2$ & $21,8 \pm 2,1 \mathrm{Aa}$ & $38,8 \pm 3,5 \mathrm{BCa}$ & $38,0 \pm 3,2 \mathrm{Ca}$ & $37,4 \pm 2,6 \mathrm{Ca}$ & $32,3 \pm 2,8 \mathrm{Ba}$ \\
\hline & G3 & $15,3 \pm 1,8 \mathrm{Aa}$ & $27,7 \pm 3,3 \mathrm{BCa}$ & $38,4 \pm 3,9 \mathrm{Ca}$ & $40,1 \pm 3,7 \mathrm{Ca}$ & $25,8 \pm 2,7 \mathrm{Ba}$ \\
\hline & G4 & $17,4 \pm 2,7 \mathrm{Aa}$ & $26,3 \pm 3,6 \mathrm{BCa}$ & $36,1 \pm 3,2 \mathrm{Ca}$ & $38,0 \pm 3,5 \mathrm{Ca}$ & $24,0 \pm 1,9 \mathrm{Ba}$ \\
\hline \multirow[t]{4}{*}{ PAM } & G1 & $51,8 \pm 3,1 \mathrm{Aa}$ & $53,3 \pm 5,2 \mathrm{Aa}$ & $57,3 \pm 3,2 \mathrm{Aa}$ & $56,6 \pm 5,6 \mathrm{Aa}$ & $59,6 \pm 7,8 \mathrm{Aa}$ \\
\hline & $\mathrm{G} 2$ & $56,8 \pm 5,3 \mathrm{Aa}$ & $60,3 \pm 3,9 \mathrm{Aa}$ & $61,1 \pm 4,9 \mathrm{Aa}$ & $64,5 \pm 3,9 \mathrm{Aa}$ & $63,5 \pm 5,9 \mathrm{Aa}$ \\
\hline & G3 & $62,6 \pm 2,0 \mathrm{Aa}$ & $63,1 \pm 2,4 \mathrm{Aa}$ & $60,8 \pm 1,6 \mathrm{Aa}$ & $61,8 \pm 2,2 \mathrm{Aa}$ & $55,9 \pm 4,1 \mathrm{Aa}$ \\
\hline & G4 & $51,2 \pm 3,7 \mathrm{Aa}$ & $55,1 \pm 2,9 \mathrm{Aa}$ & $54,9 \pm 1,4 \mathrm{Aa}$ & $62,8 \pm 1,9 \mathrm{Aa}$ & $50,3 \pm 4,6 \mathrm{Ea}$ \\
\hline \multirow[t]{4}{*}{$\mathrm{T}$} & G1 & $37,8 \pm 0,2 \mathrm{Aa}$ & $37,3 \pm 0,2 \mathrm{Ba}$ & $36,9 \pm 0,2 \mathrm{Ca}$ & $36,6 \pm 0,2 \mathrm{Da}$ & $36,1 \pm 0,2 \mathrm{Ea}$ \\
\hline & $\mathrm{G} 2$ & $38,3 \pm 0,2 \mathrm{Aa}$ & $37,6 \pm 0,2 \mathrm{Ba}$ & $36,9 \pm 0,2 \mathrm{Ca}$ & $36,6 \pm 0,3 \mathrm{Da}$ & $36,2 \pm 0,3 \mathrm{Ea}$ \\
\hline & G3 & $38,2 \pm 0,1 \mathrm{Aa}$ & $37,9 \pm 0,1 \mathrm{Ba}$ & $37,2 \pm 0,1 \mathrm{Ca}$ & $36,6 \pm 0,2 \mathrm{Da}$ & $36,3 \pm 0,2 \mathrm{Ea}$ \\
\hline & G4 & $38,2 \pm 0,2 \mathrm{Aa}$ & $38,0 \pm 0,1 \mathrm{Ba}$ & $37,5 \pm 0,1 \mathrm{Ca}$ & $37,0 \pm 0,1 \mathrm{Da}$ & $36,5 \pm 0,1 \mathrm{Ea}$ \\
\hline \multirow[t]{4}{*}{$\mathrm{pH}$} & G1 & $7,42 \pm 0,02 \mathrm{Aa}$ & $7,31 \pm 0,01 \mathrm{Ba}$ & $7,30 \pm 0,02 \mathrm{BCa}$ & $7,26 \pm 0,03 \mathrm{Ca}$ & $7,28 \pm 0,03 \mathrm{BCa}$ \\
\hline & G2 & $7,44 \pm 0,01 \mathrm{Aa}$ & $7,35 \pm 0,02 \mathrm{Ba}$ & $7,29 \pm 0,02 \mathrm{BCa}$ & $7,28 \pm 0,03 \mathrm{Ca}$ & $7,29 \pm 0,04 \mathrm{BCa}$ \\
\hline & G3 & $7,41 \pm 0,01 \mathrm{Aa}$ & $7,34 \pm 0,01 \mathrm{Ba}$ & $7,35 \pm 0,02 \mathrm{BCa}$ & $7,33 \pm 0,02 \mathrm{Ca}$ & $7,34 \pm 0,02 \mathrm{BCa}$ \\
\hline & G4 & $7,39 \pm 0,01 \mathrm{Aa}$ & $7,32 \pm 0,01 \mathrm{Ba}$ & $7,28 \pm 0,01 \mathrm{BCa}$ & $7,24 \pm 0,02 \mathrm{Ca}$ & $7,29 \pm 0,03 \mathrm{BCa}$ \\
\hline \multirow[t]{4}{*}{$\mathrm{PaO}_{2}$} & G1 & $89,5 \pm 11 \mathrm{Aa}$ & $240 \pm 22 \mathrm{Ba}$ & $269 \pm 26 \mathrm{Ba}$ & $260 \pm 28 \mathrm{Ba}$ & $249 \pm 28 \mathrm{Ba}$ \\
\hline & G2 & $89,4 \pm 3,8 \mathrm{Aa}$ & $239 \pm 19 \mathrm{Ba}$ & $255 \pm 18 \mathrm{Ba}$ & $281 \pm 20 \mathrm{Ba}$ & $284 \pm 19 \mathrm{Ba}$ \\
\hline & G3 & $91,5 \pm 4,6 \mathrm{Aa}$ & $232 \pm 24 \mathrm{Ba}$ & $208 \pm 21 \mathrm{Ba}$ & $203 \pm 21 \mathrm{Ba}$ & $199 \pm 23 \mathrm{Ba}$ \\
\hline & G4 & $73,2 \pm 3,9 \mathrm{Aa}$ & $220 \pm 17 \mathrm{Ba}$ & $174 \pm 19 \mathrm{Ba}$ & $151 \pm 18 \mathrm{Ba}$ & $164 \pm 22 \mathrm{Ba}$ \\
\hline \multirow[t]{4}{*}{$\mathrm{PaCO}_{2}$} & G1 & $40,0 \pm 2,3 \mathrm{Aa}$ & $59,5 \pm 2,3 \mathrm{Aa}$ & $63,4 \pm 3,1 \mathrm{Ba}$ & $73,1 \pm 5,4 \mathrm{Ba}$ & $70,8 \pm 5,9 \mathrm{Ba}$ \\
\hline & $\mathrm{G} 2$ & $44,1 \pm 1,3 \mathrm{Ab}$ & $52,1 \pm 2,2 \mathrm{Ab}$ & $64,0 \pm 3,8 \mathrm{Bb}$ & $70,4 \pm 5,5 \mathrm{Bb}$ & $70,4 \pm 6,7 \mathrm{Bb}$ \\
\hline & G3 & $40,2 \pm 1,6 \mathrm{Aa}$ & $52,2 \pm 1,6 \mathrm{Aa}$ & $52,1 \pm 2,5 \mathrm{Ba}$ & $55,3 \pm 2,9 \mathrm{Ba}$ & $54,6 \pm 3,9 \mathrm{Ba}$ \\
\hline & G4 & $44,0 \pm 1,2 \mathrm{Ab}$ & $55,8 \pm 1,2 \mathrm{Ab}$ & $65,6 \pm 3,0 \mathrm{Bb}$ & $74,1 \pm 4,9 \mathrm{Bb}$ & $66,9 \pm 6,2 \mathrm{Bb}$ \\
\hline \multirow[t]{4}{*}{$\mathrm{cHCO}_{3}$} & G1 & $25,2 \pm 1,1 \mathrm{Aa}$ & $29,0 \pm 0,6 \mathrm{Aa}$ & $30,4 \pm 0,6 \mathrm{Ba}$ & $31,3 \pm 0,7 \mathrm{Ba}$ & $31,2 \pm 0,9 \mathrm{Ba}$ \\
\hline & $\mathrm{G} 2$ & $25,6 \pm 0,6 \mathrm{Aa}$ & $27,9 \pm 0,8 \mathrm{Aa}$ & $29,8 \pm 0,7 \mathrm{Ba}$ & $30,8 \pm 0,8 \mathrm{Ba}$ & $31,5 \pm 0,9 \mathrm{Ba}$ \\
\hline & G3 & $34,6 \pm 0,6 \mathrm{Aa}$ & $27,4 \pm 0,6 \mathrm{Aa}$ & $27,4 \pm 0,6 \mathrm{Ba}$ & $28,3 \pm 0,7 \mathrm{Ba}$ & $28,1 \pm 0,9 \mathrm{Ba}$ \\
\hline & G4 & $25,5 \pm 0,6 \mathrm{Aa}$ & $27,7 \pm 0,3 \mathrm{Aa}$ & $29,4 \pm 0,5 \mathrm{Ba}$ & $30,3 \pm 0,5 \mathrm{Ba}$ & $29,9 \pm 0,6 \mathrm{Ba}$ \\
\hline
\end{tabular}

Potencial de hidrógeno $(\mathrm{pH})$, presión parcial de oxígeno en $\mathrm{mmHg}\left(\mathrm{PaO}_{2}\right)$, presión parcial de dióxido de carbono en $\mathrm{mmHg}\left(\mathrm{PaCO}_{2}\right)$, concentración de bicarbonato en $\mathrm{mmol} / \mathrm{L}\left(\mathrm{CHCO}_{3}\right)$, frecuencia cardiaca en lat $/ \mathrm{min}(\mathrm{FC})$, frecuencia respiratoria ciclos/min (FR) presión arterial media en $\mathrm{mmHg}$ (PAM) y temperatura rectal en ${ }^{\circ} \mathrm{C}(\mathrm{T})$. Letras distintas indican diferencia estadística $(\mathrm{P}<0,05)$, siendo mayúsculas entre los momentos de evaluación y minúsculas entre los grupos de tratamiento.

dos factores: primero, la absorción del $\mathrm{CO}_{2}$ utilizado para inducir el neumoperitoneo y, segundo, la alteración en la relación ventilación-perfusión pulmonar causada por la compresión del diafragma (Hayden y Cowman 2011), resultando en el aumento de la $\mathrm{PaCO}_{2}$ que induce acidosis respiratoria y, consecuentemente, elevación del bicarbonato sanguíneo por la activación de factores compensatorios, en la tentativa de mantener el equilibrio ácido básico (Safran y Orlando 1994).

$\mathrm{La} \mathrm{PaCO}_{2}$, por otro lado, también evidenció alteraciones relacionadas con el tratamiento opioide, mostrándose mayor $(P=0,02)$ en los animales tratados con butorfanol 
(cuadro 1). La elevación de la $\mathrm{PaCO}_{2}$ (hipercapnia) es consecuencia directa de la depresión respiratoria, comúnmente causada por los fármacos opioides en animales (Clarke y col 2014). Esta alteración inducida por el butorfanol ya fue descrita en ovejas sometidas a anestesia disociativa a las mismas dosis utilizadas en este estudio (Howard y col 1990); sin embargo Waterman y col (1991) y Dzikiti y col (2014) no relataron alteraciones respiratorias en ovejas y cabras como efectos directos del butorfanol en dosis más bajas, haciendo pensar que este efecto sea dependiente de la alta dosis utilizada.

La temperatura corporal disminuyó significativamente $(\mathrm{P}<0,01)$ durante el procedimiento, los animales perdieron en promedio $2{ }^{\circ} \mathrm{C}$ (cuadro 1), siendo esta alteración común en procedimientos quirúrgicos (Biazzotto et al 2006). El tiempo de recuperación anestésica fue de 56,2 $\pm 2,9$ minutos, siendo similar en todos los grupos estudiados. El protocolo anestésico utilizado ocasiona un tiempo de recuperación prolongado cuando se compara con otras asociaciones farmacológicas utilizadas para la anestesia general en caprinos (Hikasa y col 1998, Dzikiti y col 2009) posiblemente debido a la hipotermia e hipotensión ocasionadas. Ninguna de las hembras presentó señales de dolor o incomodidad durante las 72 horas de observación, por tal razón, ningún tratamiento de rescate analgésico requirió ser administrado; de esta forma se corrobora la afirmación de que la LOPU es poco dolorosa en pequeños rumiantes (Malm y col 2004, Teixeira y col 2011). Por su parte, y aunque, el butorfanol en dosis de 0,05 a $0,4 \mathrm{mg} /$ $\mathrm{kg}$ presenta una actividad antinociceptiva comprobada en ovejas (Waterman y col 1991), en este estudio fue utilizada una dosis superior intentando proporcionar una analgesia profunda y evaluar sus repercusiones cardiorrespiratorias, sin embargo no fue evidenciada diferencia en el dolor posoperatorio durante las evaluaciones realizadas y sería recomendable un estudio más profundo de su efecto analgésico en este tipo de procedimientos.

En las condiciones estudiadas con este protocolo anestésico se observaron un tiempo de recuperación prolongado, hipotensión clínica y acidosis respiratoria; la suplementación con butorfanol resultó en aumento de la $\mathrm{PaCO}_{2}$ sin mejora evidente de la analgesia posoperatoria. Por estos motivos, la aplicación práctica de este protocolo en cabras que requieran ser sometidas a cirugía laparoscópica indica un cuidado especial de la fluidoterapia, la ventilación y la recuperación; siendo poco recomendable. Estudios más profundos del efecto analgésico del butorfanol en estas situaciones se hacen necesarios para permitir indicar su utilización.

\section{AGRADECIMIENTOS}

A la Fundación de Amparo a la Investigación del Estado de São Paulo (FAPESP), al Consejo Nacional de Desenvolvimiento Científico y Tecnológico (CNPq) y a la Coordinación de Perfeccionamiento del Personal de Nivel Superior (CAPES) por el apoyo financiero y las becas estudiantiles concebidas.

\section{REFERENCIAS}

Afshar FS, A Baniadam, SP Marashipour. 2005. Effect of xylazineketamine on arterial blood pressure, arterial blood ph, blood gases, rectal temperature, heart and respiratory rates in goats. Bull Vet Inst Pulawy 49, 481-484.

Bardoczky GI, E Engelman, M Levarlet, P Simon. 1993. Ventilatory effects of pneumoperitoneum monitored with continuous spirometry. Anesthesia 48, 309-311.

Biazzotto CB, M Brudniewski, AP Schmidt, JOCA Junior. 2006. Hipotermia no período perioperatório. Revista Brasileira Anestesiologia 56, 89-106.

Carroll GL, RN Hooper, MR Slater, SM Hartsfield, NS Matthews. 1998. Detomidine-butorphanol-propofol for carotid artery translocation and castration or ovariectomy in goats. Vet Surg 27, 75-82.

Celly CS, WN McDonell, SS Young, WD Black. 1997. The comparative hypoxaemic effect of four a adrenoceptor agonists (xylazine, romifidine, detomidine and medetomidine) in sheep. J Vet Pharmacol Therap 20, 464-471.

Clarke KW, CM Trim, LW Hall. 2014. Veterinary Anaesthesia. $11^{\mathrm{a}} \mathrm{ed}$. Elsevier, London, UK.

Dzikiti TB, GF Stegmann, LJ Hellebrekers, RE Auer, LN Dzikiti. 2009. Sedative and cardiopulmonary effects of acepromazine, midazolam, butorphanol, acepromazine-butorphanol and midazolam-butorphanol on propofol anaesthesia in goats. J S Afr Vet Assoc 80, 10-16.

Dzikiti T, GE Zeiler, LN Dzikiti, ER Garcia. 2014. The effects of midazolam and butorphanol, administered alone or combined, on the dose and quality of anaesthetic induction with alfaxalone in goats. J S Afr Vet Assoc 85, 1.

Fahy BG, GM Barnas, SE Nagle, JL Flowers, MJ Njoku, M Agarwal. 1996. Changes in lung and chest wall properties with abdominal insufflation of cabon dioxide are immediately reversible. Anesth Analg 82, 501-505.

Güzel Ö, D Olgun Erdikmen, D Aydin, Z Mutlu, E Yildar. 2012. Investigation of the eff ects of $\mathrm{CO} 2$ insufflation on blood gas values during laparoscopic procedures in pigs. Turk $J$ Vet Anim Sci 36, 183-187.

Hayden P, S Cowman. 2011. Anaesthesia for laparoscopic surgery. Contin Educ Anaesth Crit Care Pain 11, 177-180.

Hikasa Y, K Okuyama, T Kakuta, K Takase, S Ogasawara. 1998. Anesthetic potency and cardiopulmonary effects of sevoflurane in goats: comparison with isoflurane and halothane. Can J Vet Res 62, 299-306.

Howard BW, MS Lagutchik, AJ Januszkiewicz, DG Martin. 1990. The cardiovascular response of sheep to tiletamine-zolazepam and butorphanol tartrate anesthesia. Vet Surg 19, 461-467.

Ivankovich AD, DJ Miletich, RF Albrecht, HJ Heyman, RF Bonnet. 1975. Cardiovascular effects of intraperitoneal insufflation with carbon dioxide and nitrous oxide in the dog. Anesthesiology 42, 281-217.

Johnston MS. 2005. Clinical approaches to analgesia in Ferrets and Rabbits. Semin Avian and Exot Pet 14, 229-235.

Kokkonen UM, L Eriksson. 1987. Cardiovascular and allied actions of xylazine and atropine in the unanaesthetized goat. J Vet Pharmacol Therap 10, 11-16.

LiJian J, HB Wang, HJ Xiong, JT Zhang, L Gao. 2008. Effects of different carbon dioxide-induced pneumoperitoneum on hepatic and renal function in goats. Chinese J Vet Sci 8, 973-977.

Malm C, PR Savassi-Rocha, VA Gheller, HP Oliveira, AR Lamounier, V Foltyneck. 2004. Ovário-histerectomia: estudo experimental comparativo entre as abordagens laparoscópicas e convencionais na espécie canina. Intra-operatório. I. Arq Bras Med Vet Zootec $56,457-466$.

O’Leary E, K Hubbard, W Tormey, AJ Cunningham. 1996. Laproscopic cholecystectomy: haemodynamic and neuroendocrine responses after pneumoperitonium and changes in position. British J Anaesth 76, 640-644.

Otero PE. 2005. O Manejo da Dor e a Medicina Veterinária. In: Otero PE (ed). Dor Avaliação e Tratamento em Pequenos Animais. Interbook, São Caetano do Sul, Brasil, Pp 2-5. 
Safran DB, R Orlando. 1994 Physiologic effect of pneumoperitoneum. Am J Surg 167, 281-286.

Schiller WR. 1987. The Trendelenburg position. Surgical aspects. In: Martin JT (ed). Positoning in Anesthesia and Surgery. $2^{\text {nd }}$ ed. WB Saunders, Philadelphia, USA, Pp 117-126.

Teixeira PPM, LC Padilha, MEF Oliveira, TF Motheo, ASL Silva, FFPC Barros, LN Coutinho, FN Flôres, MCS Lopes, MB Bandarra, WRR
Vicente. 2011. Aspiração folicular por videolaparoscopia em ovelhas santa inês: descrições da técnica. Rev Cient Elet Med Vet 16, 1-13.

Tekin A, C Evruke, O Deveci. 2009. Maternal and fetal near-term sheep cytokine responses to carbon dioxide pneumoperitoneum. Surg Laparosc Endosc Percutan Tech 19,138-141.

Waterman AE, A Livingston, A Amin. 1991. Analgesic activity and respiratory effects of butorphanol in sheep. Res Vet Sci 51, 19-23. 